\title{
COMPARATIVE STUDY ON DIFFERENT PHYSICAL PROPERTIES OF GREY \& FINISHED SINGLE JERSEY DERIVATIVES
}

\author{
Suraiya Ireen and Md. Mahbubur Rahman
}

\begin{abstract}
Knitting is the mostly used fabric manufacturing processes in Bangladesh through which various types of knitted fabrics with different physical and chemical properties are produced. Single jersey derivatives have some dimensional, physical or chemical properties that change according to some factors and processing. These properties also change after dyeing \& finishing process. As finishing is mandatory for fabric production, some tests are carried out after finishing stage \& proper controlling is done according desired quality. Single jersey fabrics show low strength and higher abrasion resistance than grey fabric after dyeing \& finishing. Finished fabric is more stiff than grey. Spirality \& shrinkage properties of the single jersey fabric are rapidly change after dyeing \& finishing and generally acceptable under the limit. Therefore, this paper is all about the comparison of physical properties among various grey and finished single jersey weft knitted fabrics which will be helpful to know for efficient production, taking corrective actions to minimize changes of basic physical properties and produce quality knit products.
\end{abstract}

Index Terms - GSM, Spirality, Shrinkage, Abrasion Resistance, Stiffness.

\section{INTRODUCTION}

In a weft knitted structure a horizontal row of loops can be made using one thread and the thread runs in the horizontal direction. Most of the knitted fabrics are produced by weft knitting which can be single jersey or double jersey [1]. Single Jersey is a weft knitted fabric produced with one set of needles is called single Jersey or plain knitted fabric. These fabrics have physical properties like lighter fabric, highly extensible in length \& width, high drapability $\&$ good dimensional stability, high shrinkage in

This paper was received on 22 April 2019, accepted on 19 November 2019. This work was supported financially by Green University of Bangladesh.

Suraiya Ireen currently is a lecturer of Textile Engineering Department of Green University of Bangladesh. E-mail: suraiya@tex.green.edu.bd.

Md. Mahbubur Rahman is a senior lecturer of Textile Engineering Department of Green University of Bangladesh. E-mail: mahbub@tex.green.edu.bd. natural fiber, non-reversible, soft \& resilience, good stretch-ability and elastic recovery. Single jersey fabrics are used for leisure wear manufacturing basically men's, women's, and kid's polo T-shirts, sports-wear.

The properties of knitted fabrics are enormously dependable on its structure. Specifically, the effect of knit structure on the bursting strength, air permeability, and bending rigidity is highly remarkable in washed fabrics [2]. Prediction of the bursting strength of a knitted fabric is difficult before testing [3]. As the bending length related with the stiffness and drape property of the fabrics, it should be taken as a consideration. Buckling behavior and puckering are also caused by these properties of the fabric [4]. Pills can be seen on the knitted fabric surface which is the result of abrasion between two surfaces. Pilling varies with the surface density and stitch length which means it can be changed during finishing or garments production [5]. Fabric shrinkage occurs commonly in weft knitted fabrics which mainly cause problems in finished items. Tension variation of yarns in knitting process and relaxation process can be the cause if this dimensional changes [6]. Spirality is one kind of distorted angle which appear as the displacement of side seam in knitted fabrics. Later this will cause a serious quality problem during garments production especially after finishing [7]. Sometimes controlling of some properties of finished fabrics are beyond our trial. In that case, analysis of fabric properties at grey stage \& finished stage and comparing these properties can help us to take various control \& corrective action when necessary.

\section{MATERIALS \& METHOD}

\section{A. Materials:}

Plain single jersey (with and without finishing), Double lacoste, Lycra terry \& Fleece were used as test specimens.

\section{B. Test Method:}

\section{GSM calculation:}

It is very easy to know the weight of the fabric by cutting the fabric with the GSM cutter. 
Pilling test:

Pilling test was done by ASTM D4970 method by a Martindale Abrasion Tester.

Bursting strength test:

Bursting test was done by ISO 13938-2-1999 method by a James Heal Bursting Tester.

Stiffness test:

Stiffness test was done by ASTM D1388 by Shirley Stiffness Tester.

Shrinkage test:

Shrinkage test is carried out by AATCC 135-1987 or ISO 6330 (60 degree Celsius, tumble dry).

Spirality test:

Spirality test is carried out by AATCC Test method $179-2004$.

\section{EXPERIMENTS}

\begin{tabular}{|c|c|c|c|}
\hline Test & KPa & $\mathbf{m m}$ & s \\
\hline 1 & 221.0 & 36.7 & 22.6 \\
\hline 2 & 212.7 & 35.5 & 21.9 \\
\hline 3 & 203.6 & 33.6 & 19.2 \\
\hline Mean & 212.4 & 35.2 & 21.2 \\
\hline
\end{tabular}

Table 01: Bursting results on grey sample of plain single jersey. (only dyeing)

\begin{tabular}{|c|c|c|c|}
\hline Test & KPa & mm & s \\
\hline 1 & 155.3 & 32.3 & 16.9 \\
\hline 2 & 149.5 & 31.4 & 15.2 \\
\hline 3 & 134.2 & 30.9 & 14.6 \\
\hline Mean & 146.3 & 31.5 & 15.5 \\
\hline
\end{tabular}

Table 02: Bursting results on finished sample of plain single jersey. (only dyeing)

\begin{tabular}{|c|c|c|c|}
\hline Test & KPa & mm & s \\
\hline 1 & 317.7 & 27.1 & 32.2 \\
\hline 2 & 310.1 & 26.3 & 31.9 \\
\hline 3 & 305.3 & 25.8 & 30.1 \\
\hline Mean & 311.0 & 26.4 & 31.4 \\
\hline
\end{tabular}

Table 03: Bursting results on grey sample of plain single jersey.

\begin{tabular}{|c|c|c|c|}
\hline Test & KPa & mm & s \\
\hline 1 & 302.1 & 33.9 & 30.6 \\
\hline 2 & 295.2 & 32.0 & 29.2 \\
\hline 3 & 280.7 & 30.8 & 28.4 \\
\hline Mean & 292.6 & 32.2 & 29.4 \\
\hline
\end{tabular}

Table 04: Bursting results on finished sample of plain single jersey.

\begin{tabular}{|l|l|l|l|}
\hline Test & KPa & $\mathbf{m m}$ & S \\
\hline 1 & 245.4 & 25.8 & 25.1 \\
\hline 2 & 238.8 & 24.9 & 24.3 \\
\hline 3 & 226.6 & 22.4 & 23.2 \\
\hline Mean & 236.9 & 24.3 & 24.2 \\
\hline
\end{tabular}

\begin{tabular}{|c|c|c|c|}
\hline Test & KPa & mm & s \\
\hline 1 & 195.1 & 31.5 & 19.9 \\
\hline 2 & 186.1 & 30.7 & 18.9 \\
\hline 3 & 178.0 & 30.4 & 18.3 \\
\hline Mean & 186.4 & 30.9 & 19.0 \\
\hline
\end{tabular}

Table no. 06: Bursting results on finished sample of double lacoste

\begin{tabular}{|l|l|l|l|}
\hline Test & KPa & $\mathbf{m m}$ & $\mathbf{s}$ \\
\hline 1 & 326.1 & 50.0 & 26.1 \\
\hline 2 & 318.3 & 49.7 & 25.0 \\
\hline 3 & 304.4 & 48.1 & 24.6 \\
\hline Mean & 316.2 & 49.2 & 25.2 \\
\hline
\end{tabular}

Table no. 07: Bursting results on grey sample of lycra terry.

\begin{tabular}{|c|c|c|c|}
\hline Test & KPa & $\mathbf{m m}$ & $\mathbf{s}$ \\
\hline 1 & 235.9 & 46.1 & 23.0 \\
\hline 2 & 226.1 & 45.9 & 23.1 \\
\hline 3 & 217.5 & 43.8 & 22.4 \\
\hline Mean & 226.5 & 45.2 & 22.8 \\
\hline
\end{tabular}

Table no. 08: Bursting results on finished sample of lycra terry.

\begin{tabular}{|l|l|l|l|}
\hline Test & KPa & $\mathbf{m m}$ & $\mathbf{~}$ \\
\hline 1 & 308.4 & 48.6 & 24.3 \\
\hline 2 & 301.2 & 47.1 & 22.9 \\
\hline 3 & 295.4 & 46.4 & 21.1 \\
\hline Mean & 301.6 & 47.3 & 22.7 \\
\hline
\end{tabular}

Table no. 09: Bursting results on grey sample of fleece.

\begin{tabular}{|c|c|c|c|}
\hline Test & KPa & $\mathbf{m m}$ & s \\
\hline 1 & 220.6 & 40.0 & 21.9 \\
\hline 2 & 211.2 & 39.3 & 21.6 \\
\hline 3 & 203.8 & 38.2 & 20.7 \\
\hline Mean & 211.8 & 39.1 & 21.4 \\
\hline
\end{tabular}

Table no. 10: Bursting results on finished sample of fleece.

According to the following figure, we can see that strength falls after finishing. If fabric is not stentered or compacted, Fabric strength falls after dyeing.

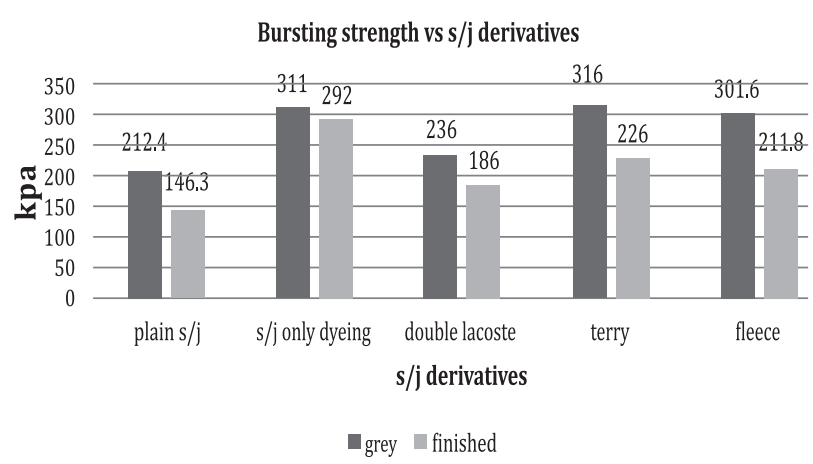

Fig no. 01: Bursting strength vs single jersey derivatives 


\begin{tabular}{|c|c|c|c|c|c|c|c|c|c|c|}
\hline \multirow{2}{*}{ Cycle } & \multicolumn{9}{|c|}{ Rating } \\
\cline { 2 - 12 } & $\begin{array}{c}\text { Plain Single jersey } \\
\text { (without finishing) }\end{array}$ & \multicolumn{2}{|c|}{$\begin{array}{c}\text { Plain Single } \\
\text { jersey }\end{array}$} & \multicolumn{2}{|l|}{ Double Lacoste } & \multicolumn{2}{|c|}{ Lycra Terry } & \multicolumn{3}{|c|}{ Fleece } \\
\cline { 2 - 12 } & Grey & Finished & Grey & Finished & Grey & Finished & Grey & Finished & Grey & Finished \\
\hline 100 & $4-5$ & $4-5$ & $4-5$ & $4-5$ & $4-5$ & $4-5$ & $3-4$ & $4-5$ & $3-4$ & $4-5$ \\
\hline 200 & $4-5$ & $4-5$ & $3-4$ & $4-5$ & $3-4$ & $4-5$ & $3-4$ & $4-5$ & $3-4$ & $3-4$ \\
\hline 300 & $3-4$ & $3-4$ & $3-4$ & $4-5$ & $3-4$ & $3-4$ & $2-3$ & $3-4$ & $3-4$ & $3-4$ \\
\hline 400 & $3-4$ & $3-4$ & $3-4$ & $3-4$ & $2-3$ & $3-4$ & $2-3$ & $3-4$ & $2-3$ & $2-3$ \\
\hline 500 & $2-3$ & $3-4$ & $3-4$ & $3-4$ & $2-3$ & $2-3$ & $2-3$ & $2-3$ & $2-3$ & $2-3$ \\
\hline 600 & $2-3$ & $2-3$ & $2-3$ & $3-4$ & $2-3$ & $2-3$ & $2-3$ & $2-3$ & $2-3$ & $2-3$ \\
\hline 700 & $2-3$ & $2-3$ & $2-3$ & $3-4$ & $2-3$ & $2-3$ & $2-3$ & $2-3$ & $2-3$ & $2-3$ \\
\hline 800 & $2-3$ & $2-3$ & $2-3$ & $2-3$ & $2-3$ & $2-3$ & $1-2$ & $2-3$ & $2-3$ & $2-3$ \\
\hline 900 & $1-2$ & $2-3$ & $2-3$ & $2-3$ & $1-2$ & $2-3$ & $1-2$ & $1-2$ & $1-2$ & $1-2$ \\
\hline 1000 & $1-2$ & $1-2$ & $1-2$ & $1-2$ & $1-2$ & $1-2$ & $1-2$ & $1-2$ & $1-2$ & $1-2$ \\
\hline
\end{tabular}

Table no.11: Pilling test results for grey \& finished sample of single jersey derivatives

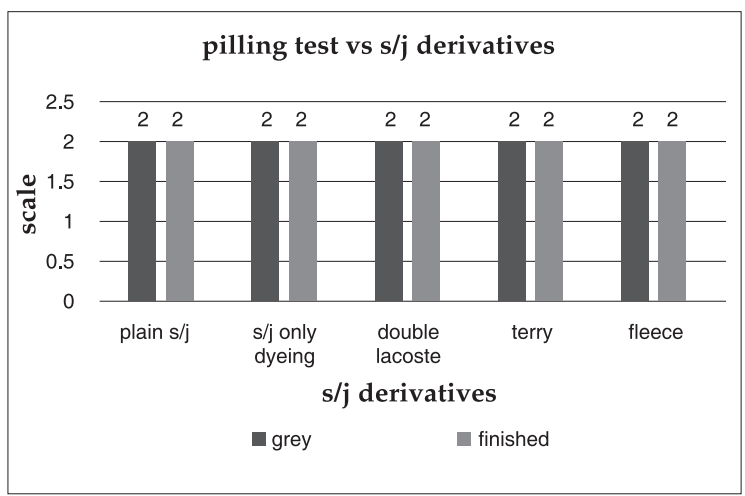

Fig no.2: Pilling test vs single jersey derivatives (after 1000-time abrasion)

According to the figure-

After 1000-time abrasion all finished fabrics show the same appearance for pilling test though before 500time abrasion some variations for grey and finished fabric according to the next figure-

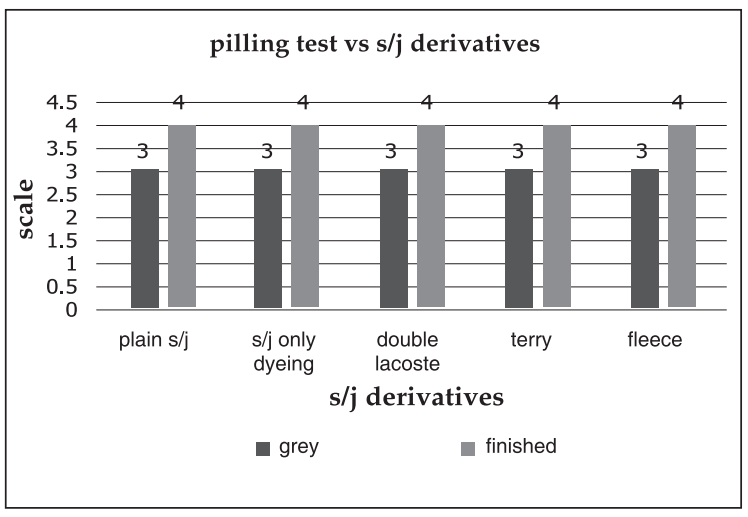

Fig no. 03: Pilling test vs single jersey derivatives (after 500-time abrasion)

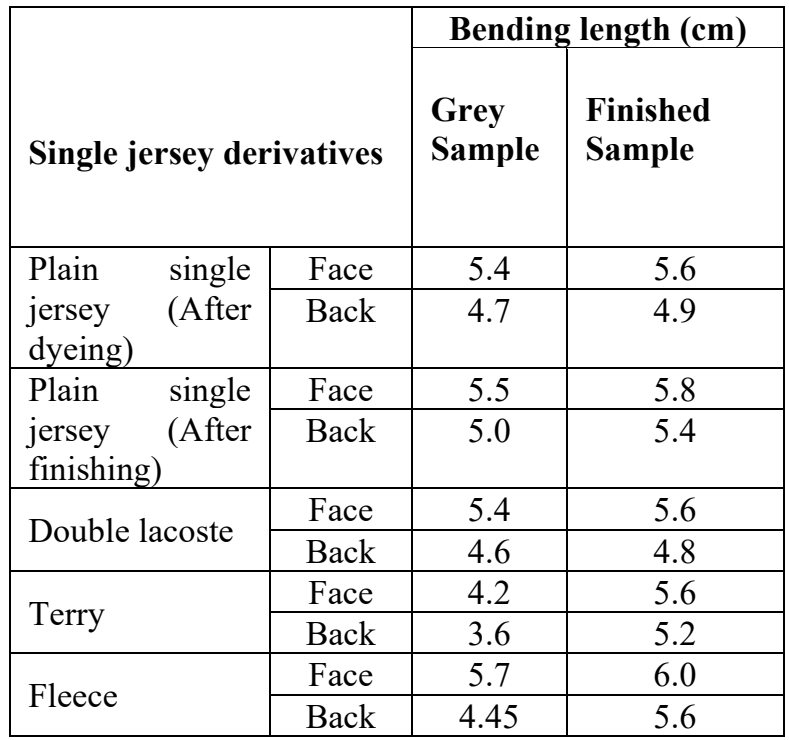

Table no. 12: Stiffness test for grey \& finished sample of single jersey derivatives

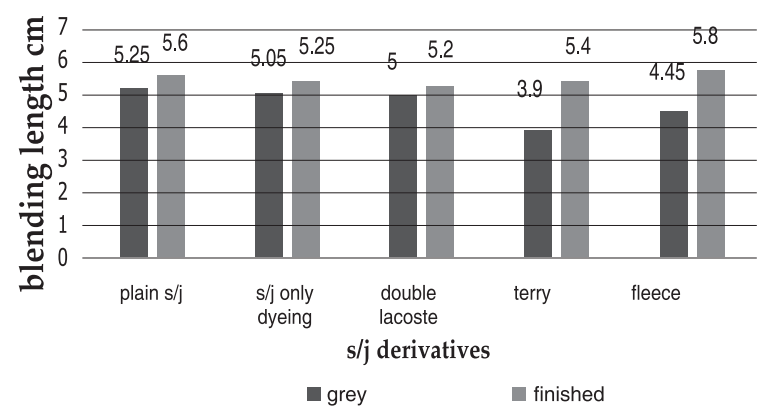

Fig no.4: Stiffness test vs single jersey derivatives

\begin{tabular}{|c|c|c|c|c|c|c|c|c|}
\hline \multirow{2}{*}{\multicolumn{2}{|c|}{$\begin{array}{c}\text { Single Jersey } \\
\text { derivatives }\end{array}$}} & \multirow{2}{*}{ Direction } & \multirow{2}{*}{$\begin{array}{l}\text { Febric length } \\
\text { before wash }\end{array}$} & \multicolumn{4}{|c|}{ Fabric length after wash } & \multirow{2}{*}{$\begin{array}{c}\text { Shrinkage } \\
\%\end{array}$} \\
\hline & & & & $X_{1}$ & $\mathrm{X}_{2}$ & $\mathrm{X}_{3}$ & Mean & \\
\hline \multirow{4}{*}{$\begin{array}{c}\text { Plain single } \\
\text { jersey (only } \\
\text { dyeing) }\end{array}$} & \multirow{2}{*}{ Grey } & Wales wise & 7 & 6.7 & 6.7 & 6.2 & 6.53 & 6.7 \\
\hline & & Course wise & 19 & 18.2 & 17.5 & 18 & 17.9 & 11 \\
\hline & \multirow{2}{*}{ Finished } & Wales wise & 6 & 5.8 & 5.6 & 6.0 & 5.8 & 3.33 \\
\hline & & Course wise & 16 & 14.7 & 14.5 & 14.5 & 14.5 & 9.3 \\
\hline \multirow{4}{*}{$\begin{array}{c}\text { Plain single } \\
\text { jersey }\end{array}$} & \multirow{2}{*}{ Grey } & Wales wise & 8 & 7.8 & 7.1 & 7.7 & 7.5 & 6.2 \\
\hline & & Course wise & 26 & 24.2 & 23.0 & 24.1 & 23.7 & 8.5 \\
\hline & \multirow{2}{*}{ Finished } & Wales wise & 5 & 5.1 & 4.9 & 4.8 & 4.9 & 2 \\
\hline & & Course wise & 20 & 19.2 & 19.2 & 19 & 19.1 & 4.5 \\
\hline \multirow{4}{*}{$\begin{array}{l}\text { Double } \\
\text { Lacoste }\end{array}$} & \multirow{2}{*}{ Grey } & Wales wise & 14 & 12.9 & 12.9 & 12.8 & 12.8 & 8.5 \\
\hline & & Course wise & 6.5 & 6 & 5.8 & 5.9 & 5.8 & 10.7 \\
\hline & \multirow{2}{*}{ Finished } & Wales wise & 12 & 11.9 & 11.9 & 12 & 11.9 & 0.83 \\
\hline & & Course wise & 6 & 5.8 & 5.9 & 5.5 & 5.7 & 5 \\
\hline \multirow{4}{*}{$\begin{array}{l}\text { Lycra } \\
\text { Terry }\end{array}$} & \multirow{2}{*}{ Grey } & Wales wise & 35 & 31.5 & 32 & 31.5 & 31.6 & 9.7 \\
\hline & & Course wise & 35 & 31.1 & 31 & 31 & 31 & 11 \\
\hline & \multirow{2}{*}{ Finished } & Wales wise & 35 & 33.2 & 33 & 33 & 33 & 5.7 \\
\hline & & Course wise & 35 & 33.9 & 34 & 33.7 & 33.8 & 3.4 \\
\hline \multirow{4}{*}{ Fleece } & \multirow{2}{*}{ Grey } & Wales wise & 12 & 10.4 & 10.8 & 10.8 & 10.66 & 11.2 \\
\hline & & Course wise & 6 & 4.8 & 5.2 & 5.8 & 4.9 & 9.6 \\
\hline & \multirow{2}{*}{ Finished } & Wales wise & 12 & 11.6 & 11.4 & 11.7 & 11.64 & 3.3 \\
\hline & & Course wise & 6 & 5.7 & 5.6 & 5.8 & 5.7 & 5 \\
\hline
\end{tabular}

Table no. 13: Shrinkage test for grey \& finished sample of single jersey derivatives 


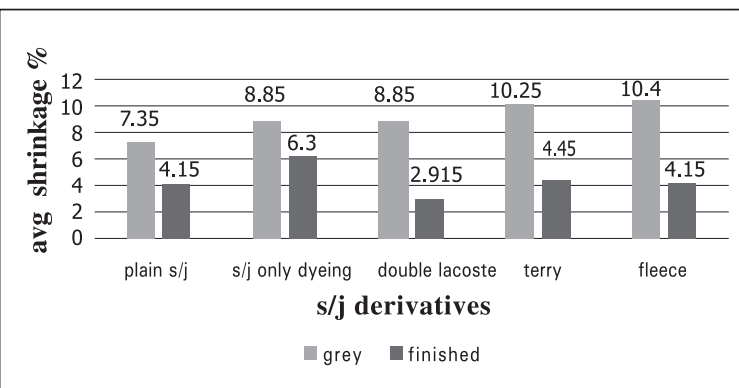

Fig no. 05: Shrinkage test vs single jersey derivatives

According to the figure, grey fabric shrinks more than finished fabric. Maximum 5\% shrinkage is acceptable though $\mathrm{s} / \mathrm{j}$ (without finishing) has more then it. So we can take a decision that most of the shrinkage decreased by stentering and compacting.

\begin{tabular}{|c|c|c|c|c|c|c|}
\hline \multirow{2}{*}{ Single jersey fabrics } & \multicolumn{3}{|c|}{ Grey sample } & \multicolumn{3}{c|}{ Finished sample } \\
\cline { 2 - 7 } & $\begin{array}{c}\mathbf{X} \\
\mathbf{c m}\end{array}$ & $\begin{array}{c}\mathbf{Y} \\
\mathbf{c m}\end{array}$ & $\tan \alpha=\mathbf{X} / \mathbf{Y}$ & $\begin{array}{c}\mathbf{X} \\
\mathbf{c m}\end{array}$ & $\begin{array}{c}\mathbf{Y} \\
\mathbf{c m}\end{array}$ & $\tan \alpha=\mathbf{X} / \mathbf{Y}$ \\
\hline Plain single jersey (only dyeing) & 0.6 & 06 & 0.10 & 0.2 & 4.5 & 0.044 \\
\hline Plain single jersey & 1.1 & 12 & 0.091 & 0.4 & 10 & 0.04 \\
\hline Double Lacoste & 0.4 & 5.5 & 0.072 & 0.0 & 06 & 0.0 \\
\hline Terry & 0.7 & 35 & 0.02 & 0.3 & 35 & 0.008 \\
\hline Fleece & 0.9 & 12 & 0.075 & 0.4 & 10 & 0.04 \\
\hline
\end{tabular}

Table no. 14: Spirality test for grey \& finished sample of single jersey derivatives

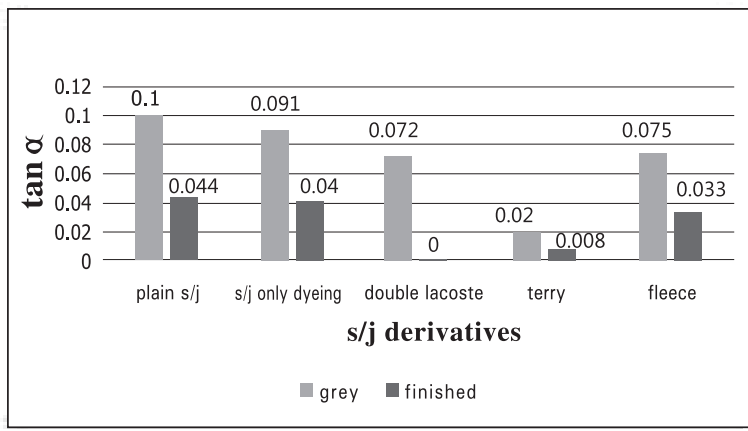

Fig no. 06: Spirality test vs single jersey derivatives

According to the figure, grey fabric has more spirality. Actually there were less knitting and dyeing fault, so spirality problem is reasonable and buyer will not claim the producer.

\begin{tabular}{|c|c|c|c|c|c|c|c|c|}
\hline Fabric name & \multicolumn{2}{|c|}{ CPI } & \multicolumn{2}{c|}{ WPI } & Stitch Density & \multicolumn{2}{c|}{ GSM } \\
\cline { 2 - 9 } & Grey & Finished & Grey & Finished & Grey & Finished & Grey & Finished \\
\hline Plain single jersey & 60 & 49 & 27 & 36 & 1620 & 1764 & 154 & 167 \\
\hline $\begin{array}{c}\text { Plain single jersey } \\
\text { (only dyeing) }\end{array}$ & 66 & 56 & 28 & 37 & 1848 & 2072 & 188 & 191 \\
\hline Double Lacoste & 80 & 84 & 24 & 23 & 1920 & 1932 & 163 & 170 \\
\hline Lycra Terry & 126 & 112 & 34 & 36 & 4284 & 4032 & 226.8 & 209 \\
\hline Fleece & 100 & 92 & 26 & 27 & 2600 & 2484 & 291.1 & 267 \\
\hline
\end{tabular}

Table no. 15: Comparison of dimensional properties among single jersey derivatives

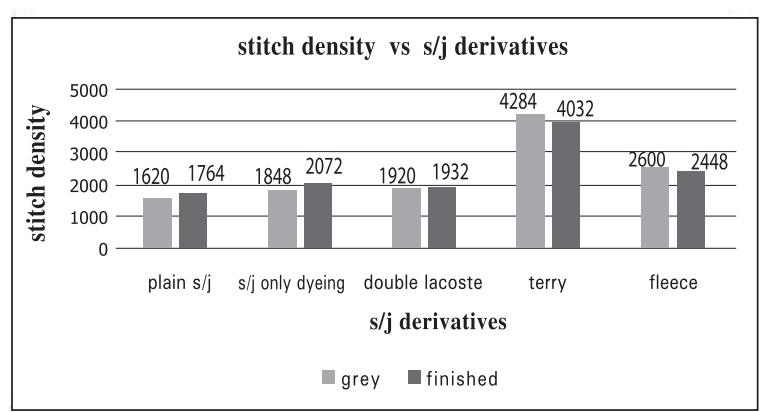

Fig no. 07: Stitch density vs single jersey derivatives

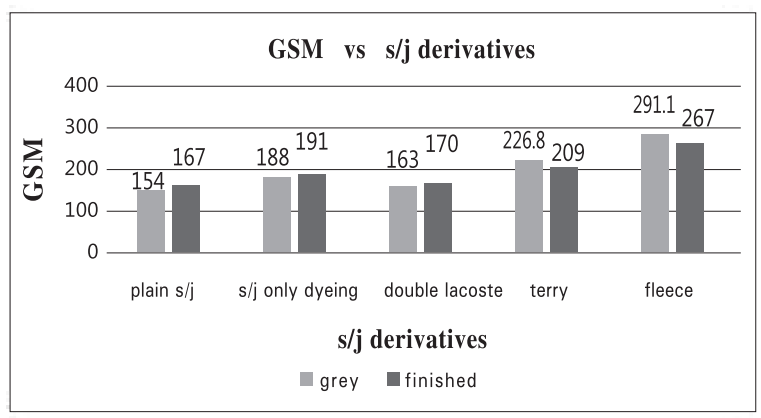

Fig no 08: GSM vs single jersey derivatives

According to the two subsequent figure, WPI,CPI,stitch density \& GSM are not constant both in grey and finished fabric. Always these parameters are higher in finished fabric than grey fabric if the fabric is remained untreated by engyme wash at the time of dyeing for removing protruding fiber.

Terry and fleece show distortion for engyme washing. For this wide variations knitting manager has to concern on finishing section since buyer does not provide grey sample. This is wrong idea that WPI increases after finishing when CPI decreases and vice versa. Heat seting is happened only when lycra is used.

Incase of terry and fleece, gsm and stitch density is higher than plain single jersey \& lacoste. Suedding may decrease the gsm and stitch density for fleece.

\section{RESUlt \& DisCUSSIONS}

\begin{tabular}{|c|c|c|c|c|c|c|}
\hline \multirow{2}{*}{ Fabric name } & \multicolumn{2}{|c|}{ Bursting test } & \multicolumn{2}{c|}{$\begin{array}{c}\text { Pilling test } \\
\text { (after } 1000 \text { time abrasion) }\end{array}$} & \multicolumn{2}{|c|}{$\begin{array}{c}\text { Stiffness test } \\
\text { average of face and back }\end{array}$} \\
\cline { 2 - 7 } & $\begin{array}{c}\text { Grey } \\
\mathrm{kpa}\end{array}$ & $\begin{array}{c}\text { Finished } \\
\mathrm{kpa}\end{array}$ & $\begin{array}{c}\text { Grey } \\
\text { scale reading }\end{array}$ & $\begin{array}{c}\text { Finished } \\
\text { scale reading }\end{array}$ & $\begin{array}{c}\text { Grey } \\
\text { cm }\end{array}$ & $\begin{array}{c}\text { Finished } \\
\text { cm }\end{array}$ \\
\hline Plain single jersey & 212.4 & 146.3 & $1-2$ & $1-2$ & 5.25 & 5.6 \\
\hline $\begin{array}{c}\text { Plain single jersey } \\
\text { (only dyeing) }\end{array}$ & 311 & 292 & $1-2$ & $1-2$ & 5.05 & 5.25 \\
\hline Double Lacoste & 236 & 186 & $1-2$ & $1-2$ & 5 & 5.2 \\
\hline Lycra Terry & 316 & 226.5 & $1-2$ & $1-2$ & 3.9 & 5.4 \\
\hline Fleece & 301.6 & 211.8 & $1-2$ & $1-2$ & 4.45 & 5.8 \\
\hline
\end{tabular}

Table no. 16: Comparison of Bursting, pilling, stiffness testing properties among single jersey derivatives 


\begin{tabular}{|c|c|l|l|l|}
\hline \multirow{2}{*}{ Fabric name } & \multicolumn{2}{|c|}{ Shrinkage test (\%) } & \multicolumn{2}{l|}{ Spirality Test (cm) } \\
\cline { 2 - 5 } & Grey & Finished & Grey & Finished \\
\hline \multirow{2}{*}{ Plain single jersey } & c-8.5 & c-4.3 & & \\
& w-6.2 & w-4 & 0.091 & 0.04 \\
\hline Plain single jersey (only dyeing) & c-6.7 & c-9.3 & & \\
& w-11 & w-3.33 & 0.091 & 0.04 \\
\hline Double Lacoste & c-10.7 & c-5 & & \\
& w-8.5 & w-.83 & 0.072 & 0.0 \\
\hline \multirow{2}{*}{ Lycra Terry } & c-11 & c-3.2 & & \\
& w-9.5 & w-5.7 & 0.02 & 0.008 \\
\hline Fleece & c-9.6 & c-5 & & \\
& w-11.2 & w-3.3 & 0.075 & 0.033 \\
\hline
\end{tabular}

Table no. 17: Comparison of Shrinkage \& spirality testing properties among single jersey derivatives

By analyzing the results, we see that the physical \& dimensional properties of fabrics change after dyeing $\&$ finishing processes. Finished fabrics show lower bursting strength than the grey fabrics. Single jersey fabrics show higher abrasion resistance which can be measured by pilling test method. After dyeing \& finishing abrasion resistance of the fabric changes because of in-between processes. Finished fabrics show higher resistance than the grey. Stiffness, spirality \& shrinkage properties of the finished sample of the single jersey fabrics vary from the grey sample. Stiffness depends on the bending properties of the fabrics. Stiffness increases after finishing because of stentering, heat setting \& other finishing processes.

\section{CONCLUSION}

Grey \& finished fabrics have distinct physical properties because of intermediary processes required to produce suitable fabrics for different applications. This analysis represents the changes of various physical properties of grey \& finished sample of single jersey derivatives because of these intermediary processes such as dyeing, compacting, stentering, heat setting etc. Generally bursting strength of finished fabric falls as well as shrinkage \& spirality which are minimized by stentering and compacting. There is no significant change in pilling but bending length will be increased after finishing. Having the knowledge of these variations should help us in the bulk production and finishing process \& also help to meet the buyer requirements. Changes of physical properties can be essential sometimes to meet required demands. However, there are some cases that are studying about the corrections or minimization of these differences and further some treatments can be originated to reduce the variations of grey and finished single jersey fabrics.

\section{Abbreviation used in this paper:}

$[\mathrm{GSM}=\mathrm{Gram}$ per square meter, WPI $=$ Wales per inch, $\mathrm{CPI}=$ Course per inch, $\mathrm{KPa}=$ Kilopascal, $\mathrm{mm}=$ Milimeter, $\mathrm{s}=$ Second]

\section{REFERENCES}

[1] D. J. Spencer, 'Knitting Technology', Third Edition, Cambridge England.

[2] Y. Kavuştura, The Effects of Some Knit Structures on the Fabric Properties in Acrylic Weft Knitted Outerwear Fabrics. 2002, pp. 40-46.

[3] S. Ertugrul, N. Ucar, 'Predicting Bursting Strength of Cotton Plain Knitted Fabrics Using Intelligent Techniques, Textile' Res. J., 2000, 70(10), pp. 845-851.

[4] T. Cassidy, C. Cassidy, S. Cassie and M. Arkison, 'The Stiffness Of Knitted Fabrics: A New Approach To The Measurement Of Bending - Part 1: Development' Leicester Polytechnic, UKEngr. International Journal of Clothing Science and Technology; ISSN: 0955-6222; Publication date: 1 May 1991

[5] Ahmed Asif, Moshiur Rahman, Farial Islam Farha, 'Effect Of Knitted Structure On The Properties Of Knitted Fabric' International Journal of Science and Research (IJSR); ISSN (Online): 2319-7064; Index Copernicus Value (2013): 6.14

[6] M. Karba, J. Gersak, Z. Stjepanovic, "The Influence of Knitting Parameters on Dimensional Changes of Knitted Fabrics in the Process of Relaxation," In Proceedings of the 2nd International Textile Clothing \& Design Conference, pp. 200-205, 2004

[7] NAE. Hassan, 'An Investigation about Spirality Angle of Cotton Single Jersey Knitted Fabrics Made from Conventional Ring and Compact Spun Yarn' Journal of American Science 2013;9(11)

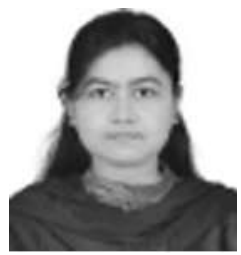

Suraiya Ireen was born in Dhaka city, in 1992. She received the B.Sc. in textile engineering from Bangladesh University of Textiles, Dhaka in 2016 and pursuing M.Sc. in textile engineering from Bangladesh University of Textiles, Dhaka. From 2016 to 2017, she worked as a Lab Executive in Epyllion Group. At present, she is working as a Lecturer in the Department of Textile Engineering, Green University of Bangladesh.

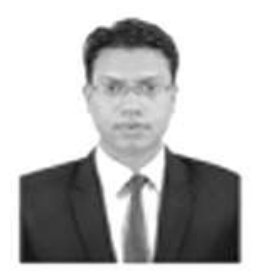

Md. Mahbubur Rahman was born in Narsingdi on 02 December 1988. At present, he is working as a Senior Lecturer in the Department of Textile Engineering, Green University of Bangladesh (GUB). He received the degree B.Sc. in Textile Engineering from Primeasia University, Dhaka, Bangladesh, in 2010 and pursuing $\mathrm{M}$. Sc in Textile Engineering from Dhaka University of Engineering \& Technology (DUET), Gazipur. His major area is Wet Processing Technology. He started his career as a production officer in Wisteria Composite Mills Ltd, Gazipur. His research interests include application of natural dyes, effects of chemicals and auxiliaries on different types of reactive dyes, effects of temperature and auxiliaries on disperse dye, effluent treatment plant (ETP) and salt-alkali free reactive dyeing. 